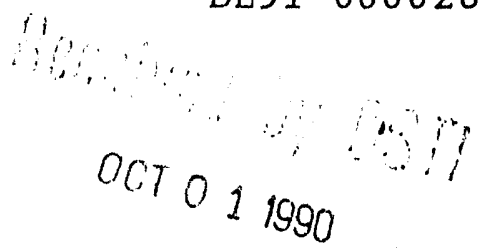

\title{
Analysis Automation With Paving: A New Quadrilateral Meshing Technique $^{\dagger}$
}

\author{
Ted D. Blacker \\ Sandia National Laboratories \\ Albuquerque, NM 87185 \\ Michael B. Stephenson \\ Scott Canann \\ Brigham Young University \\ Provo, UT 84602
}

\section{DISCLAIMER}

This report was prepared as an account of work sponsored by an agency of the United States This report was prepared as an account of work sponsored by an agency of the Uner and the Government. Neither the United States Government nor any agency thereof, nor any of their employees, makes any warranty, express or implied, or assumes any legal habs, or usefulness of any information, apparatus, product, or bility for the accuracy, completeness, or usefulness of any information, apparatus, product, or process disclosed, or represents that its use would not infringe privately owned rights. Reference herein to any specific commercial product, process, or service by trade name, trademark, manufacturer, or otherwise does not necessarily constitute or imply its endorsement, recommendation or favoring by the United States Government or any agency thereof. The views and opinions of authors expressed herein do not necessarily state or reflect those of ine United States Government or any agency thereof.

T'This work was performed at Sandia National Laboratories and supported by the U.S. Department of Energy under contract DE-ACO476 UP 00789 .

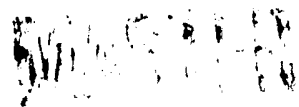




\begin{abstract}
This paper describes the impact of paving, a new automatic mesh generation algorithm, on the analysis portion of the design process. Paving generates an allquadrilateral mesh in arbitrary $2 \mathrm{D}$ geometries. The paving techuique significantly impacts the analysis process by drastically reducing the time and expertise requirements of traditional mesh generation. Paving produces a high quality mesh based on geometric boundary defunitions and user specified element sizing constraints. In this paper we describe the paving algorithm, discuss varying aspects of the impact of the technique on design automation, and elaborale on current research into 3D all-hexahedral mesh generation.
\end{abstract}




\section{Introduction}

One of the critical steps in any design methodology is a performance evaluation of the design of a part in an environment it is likely to experience. Engineering analysis has long been used to simulate an environment and predict responses of the part to that environment. Numeric computer simulations are often used to assist in the engineering analysis task. The numeric technique of finite element analysis has become a keystone for many industries is the design and understanding of complex mechanisms and processes.

Although powerful and general, the finite element technique still suffers from a lack of automation. In particular, the generation of the mesh (discretization of the geometry of the problem) is a labor and expertise intensive task. Since automation of mesh generation has a potentially large payoff, extensive research continues in this area. Most of the research focuses on techniques such as the 2D quadtree and 3D octree (Baehmann et al, 1987, Kela et al., 1986, Shepard and Yerry, 1983), triangulation (Joe and Simpson, 1986, Wordenweher, 1984) or substructuring (Schoofs et al., 1979). In general, these techniques generate meshes using triangular elements that are less accurale and less versatile than quadrilateral elements. Although some of these techniques have been extended to produce all-quadrilateral meshes, undesirable artifacts of the meshing algorithms hinder their use.

The paving technique was developed to address the analysts' needs for an automatically generated, well-formed quadrilateral mesh in arbitrary geometries. Attributes of the paving technique which distinguish it from other approaches include:

- Boundary Sensitivity. Mesh contours closely follow the contours of the boundary. This characteristic is of particular inportance since well shaped elements are usually desirable near the boundary.

- Orientation Insensitivity. Rotating or translating a given geometry does not change the resulting mesh topol- 
ogy. A mesh generated in a transformed geometry is equivalent to the original mesh transformed.

- Few Irregular Nodes. An interior mesh node in an allquadrilateral mesh is considered irregular if more or less than four elements are connected to it. This is a critical mesh topological feature because the number of elements sharing a node controls the final shape of the elements, even after smoothing. Thus a mesh with few irregular nodes, especially near the boundary where element shape is critical, is often preferred.

- Transistion Ease. Transitioning between elements of varying sizes is crucial for many finite element problems. Sinall elements must be placed in regions of large stress gradients to avoid excessive discretization errors, while larger elements are more economical in areas of low gradient or where errors are less critical. Transitioning, of necessity, introduces more irregular nodes.

- Extensibility. Extending paving to quadrilateral meshing of general 3D trimmed surfaces (3D shell elements) is a simple step and extending it to all-hexahedral mesh generation of general 3D solids, although nontrivial, appears promising.

In this paper we describe the paving algorithm and then discuss the impact this technique has on improving design and analysis productivity. This discussion includes paving's impact on initial inesh generation including 2D and a large class of 3D hexahedral mesh generation tasks, and application of the technique to adaptive analysis and large deformation analysis remeshing. We then elaborale on current research into an extension of the technique to $3 \mathrm{D}$ shell element and $3 \mathrm{D}$ all-hexahedral element niesh generation. 


\section{Paving}

The paving technique is based on iteratively layering rows of elements along the interior of a region's boundary. As shown in the brief sequence in Figure 1, these rows eventually fill the region from the boundary inward. As rows begin to overlap or coincicle at the interior of the geometry, they are carefully comnected together to form a valid quadrilateral mesh. In this section we first define the terminology and the input requirements for paving, second give an overview of each of the paving steps, and third briefly describe how these steps interact (i.e. the control sequence) during the paving process.
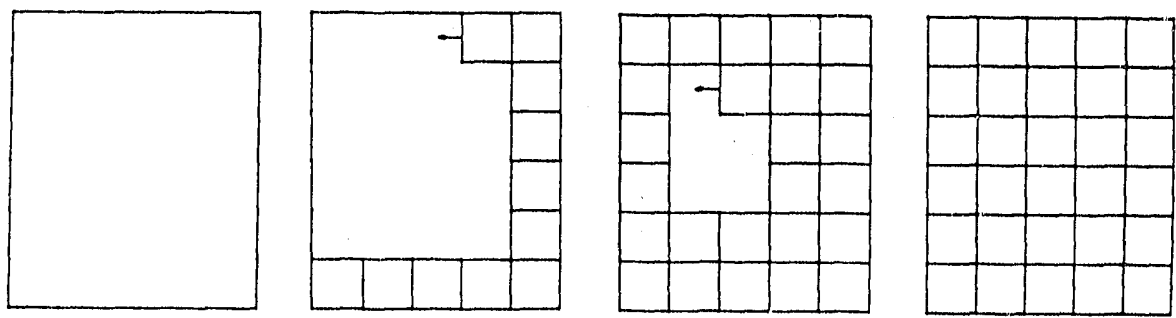

Figure 1: Example Of A Simple Paving Sequence

\section{Terminology And Input Requirements}

Paving begins with the input of one or more ordered, closed loops of connected nodes that, as shown in Figure 2, form the bounclary of the region to be meshed. 'These initial loops will be referred to as the permanent boundary of the region. The connectivity and location of nodes on the permanent boundary are not allowed to change during paving. This ensures mesh compatibility with adjacent regions.

Permanent boundaries are categorized as either exterior or interior boundaries. There is always only one exterior permanent boundary for a region. It must be a loop of nodes that is nonintersecting and completely encloses the region to be meshed. It is ordered countercluckwise. Interior permanent boundaries 


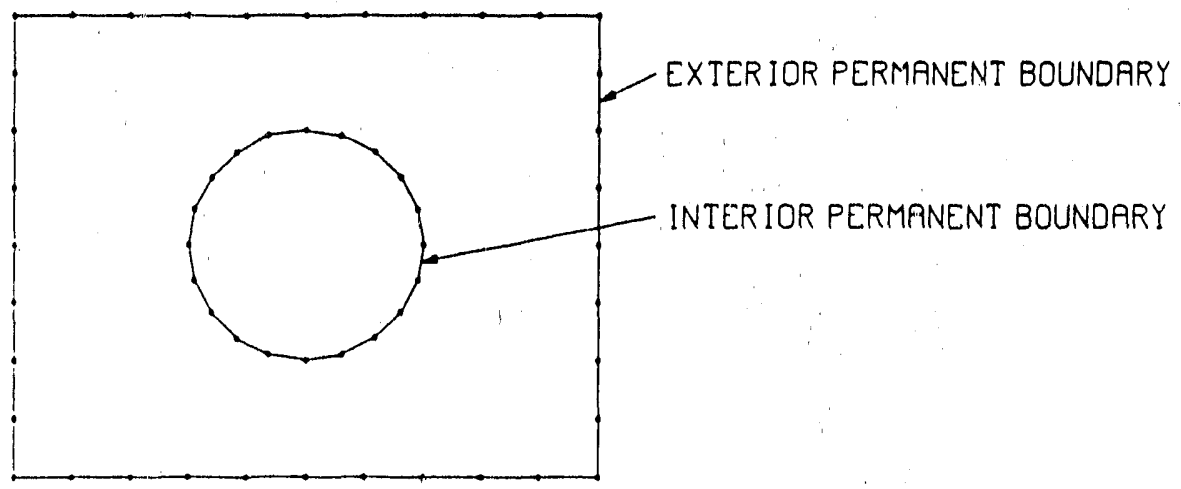

Figure 2: Example of the Permanent Boundary Input for Paving

define voids or holes in the region being meshed. They are always ordered clockwise. There may be any number or shape of interior permanent boundaries but each must be completely enclosed within the exterior permanent boundary. No permanent boundary may intersect any other permanent boundary.

During the mesh generation process, the paving technique always operates on boundaries of connected nodes referred to as paving boundaries. The paving boundaries are transient in nalure and progress as the mesh is generated. As shown in Figure 3, the paving boundaries (highlighted in bold lines) always bound the existing mesh. Initially each paving boundary is identical to a permanent boundary. Elements that are added to the mesh eventually separate the paving boundaries from their respective permanent boundaries.

Each paving boundary must always contain an even number of nodes. This is a necessary condition when generating an allquadrilateral mesh. This condition is enforced by controlling the operations performed during paving.

The size of the elements in the mesh is determined by the spacing of nodes on the paving boundary as it propagates. The spacing on a paving boundary is, of course, initially defined by the fixed node spacings on the corresponding exterior boundary. Every atlempt is made to maintain this spacing or size as the boundary progresses. Thus, the user can control the element 


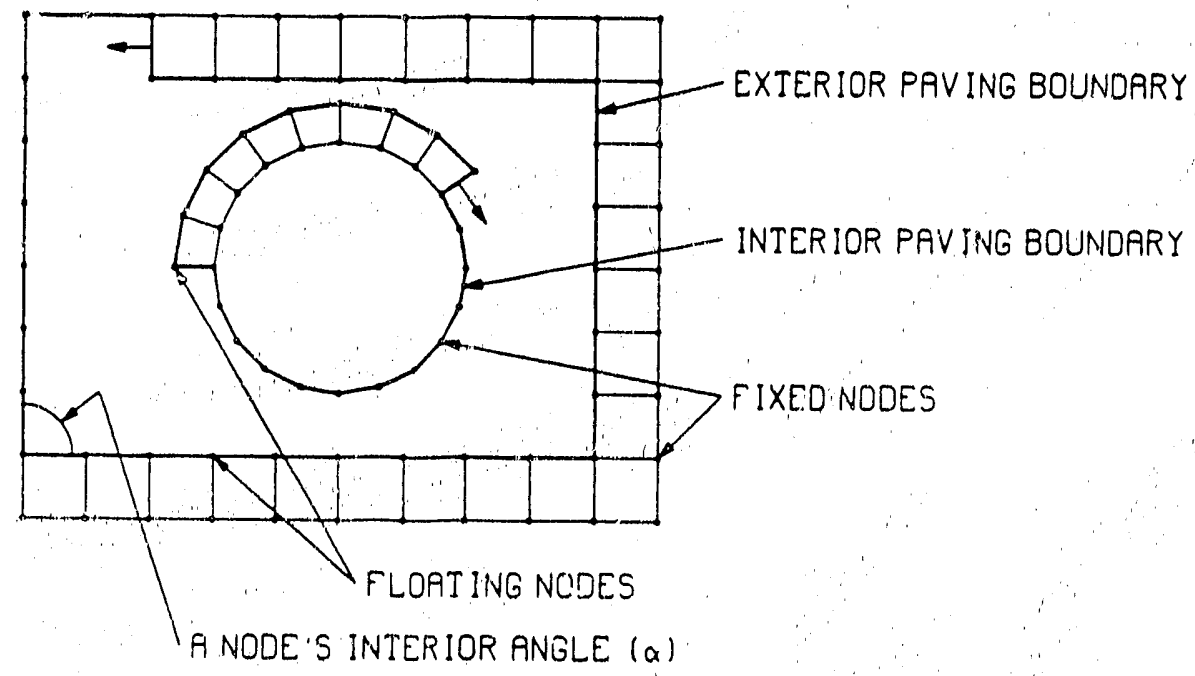

Figure 3: Example of Interior and Exterior Paving Boundaries

size by modifying the spacing of initial nodes on a permanent boundary. This spacing need not be uniform and frequently is not as it is often desirable to mesh a region with a gradation of element sizes.

\section{Overview of Paving Steps}

The propagation of a paving boundary involves a number of operations which must be tightly controlled to ensure mesh validity and quality. Each of the paving operations are explained in detail in Blacker and Stephenson (1990), but may be summarized as follows:

1. Row Choice. The beginning and ending node of the next sequence or row of elements to be added is found.

2. Closure Check. A check is made to ensure that more than six modes remain in the paving boundary. Specific closure techniques are used to conclude meshing for paving boundaries of six or fewer nodes. 
3. Row Generation. The next row of elements identified in the row choice step is incrementally added to the boundary.

4. Smooth. The position of nodes is adjusted to improve mesh quality and paving boundary smoothness.

5. Seam. Small interior angles in the paving boundary are seamed or closed by connecting opposing elements.

6. Row Adjustment. Adjustment operations are performed on the new row to correct for elements becoming too sinall or too large.

7. Intersection. The paving boundary is checked for intersections with itself or with other paving boundaries. Intersections are comnected to form new, often separate paving boundaries.

8. Cleanup. The completed inesh is adjusted where element deletion and/or addition improves the overall mesh quality.

\section{Control Sequence}

The paving process is an iterative method which uses the steps outlined above. The control of these steps is critical to the formation of a high quality mesh. The algorithm used for paving is given in Figure 4.

As can be seen in Figure 4, these steps sometimes interact in complex ways. For instance, during a row generation, it may be necessary to suspend creation of the row at critical points, smooth, look for seams and intersections, and then continue with the row generation if appropriate. Also, whenever an intersection occurs, the mesh is smoothed and the boundary is seamed on both sides before continuing to the choice of the next row. Iteration continues until all paving boundaries close, thus completing the mesh. 


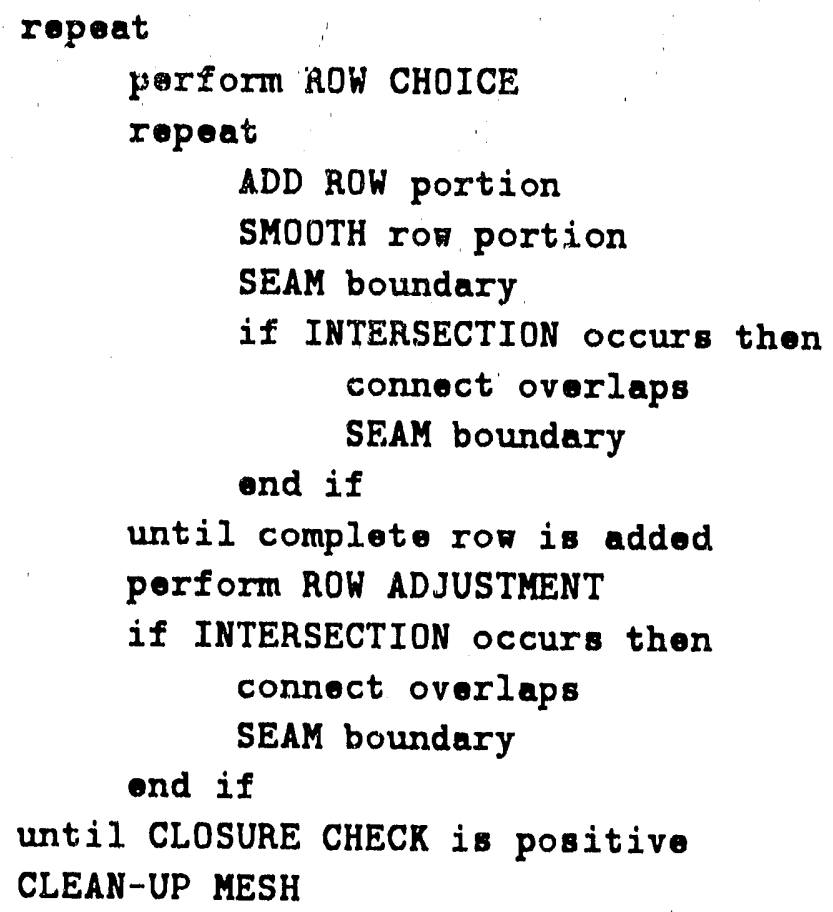

Figure 4: Paving Control Algorithm Psuedo-Code 


\section{Paving Example}

The paving process is best illustrated by an animated sequence of meshes showing how the process proceeds. Figure 5 shows such a sequence for a region with two interior slots. This geometry is further complicated by the fact that the boundary does not have equally spaced intervals. The upper slot has a relatively fine spacing of nodes as compared to the exterior boundary while the lower slot has intervals more nearly the size of the exterior boundary intervals. The upper right curved portion of the exterior boundary also has a rlatively fine resolution. As the sequence progresses, the paving boundary propogating outward from the upper slot eventually overlaps the exterior paving boundary propogating inward, forcing adjustments to be made to accommodate unequal mesh sizes. These boundaries are successfully connected (upper right frame), reducing the number of paving boundaries from three to two. Meshing continues in a straightforward fashion until it becomes evident that the element sizes in the rows around the lower slot are becoming unacceptably large (center row, left frame). The adjustments that are then made to the outer row to curtail the expansion (center frame) also cause the two paving boundaries to overlap. These are connected (center row, right frame) and meshing continues until the entire geometry is appropriately filled with elements (bottom row). Although this example does not show any extreme transitions, it does show the generality of the technique to handle irregular geometries and sizing constraints. 


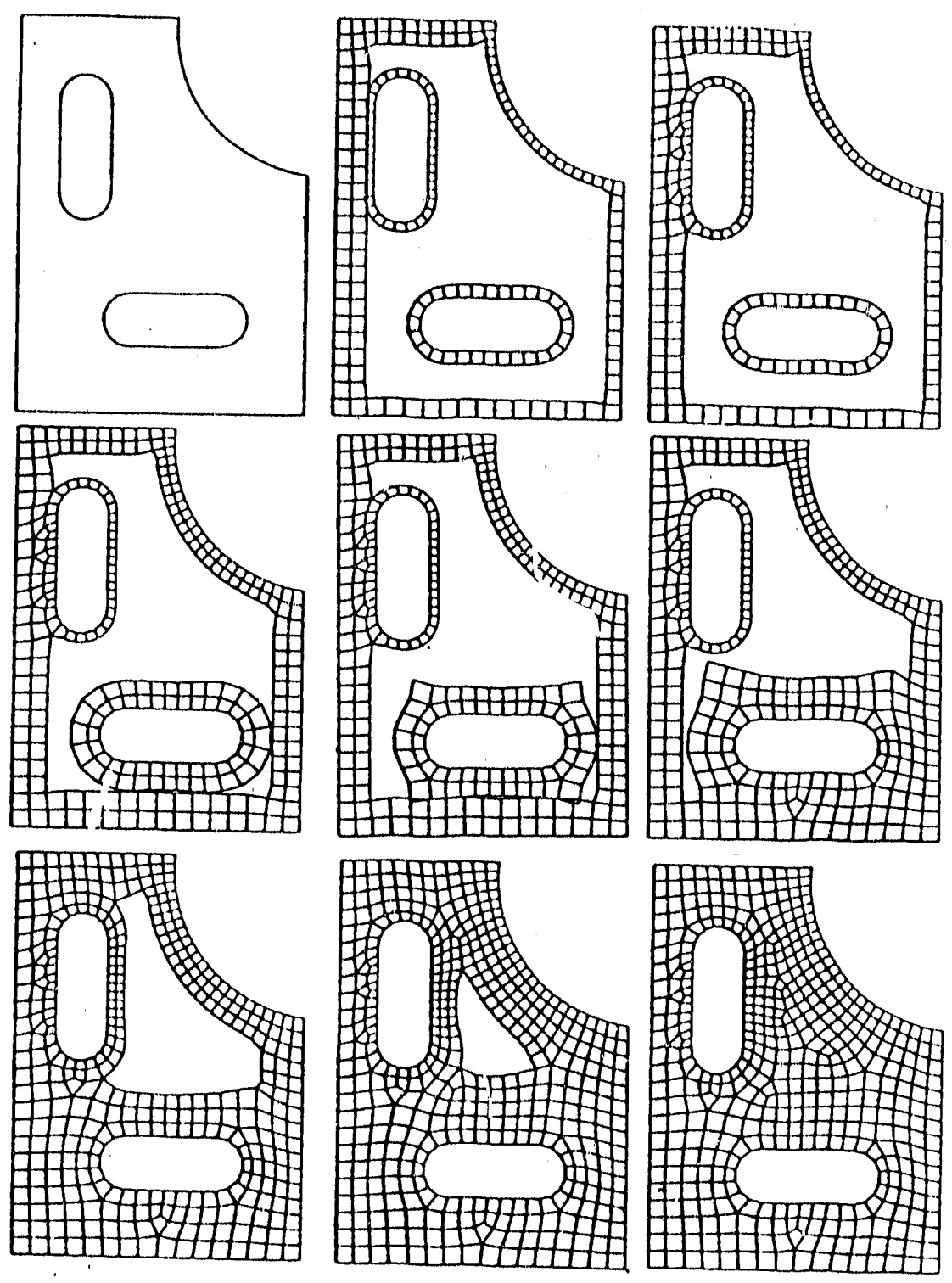

- Figure 5: Example Of A Complex Paving Sequence 


\section{The Impact of Paving on Productivity}

As mentioned earlier, the paving algorithm substantially automates mesh generation, one of the most time consuming of tasks for design analysis. This translates to savings not only during the generation of a mesh to fit an arbitrary 2D geometry, but also impacts some of the more exacting tasks the analyst may encounter during the analysis process. This section will elaborate on the impact of the technique on these tasks including the initial 2D mesh generation, 3D all-hexahedral mesh generation for a large class of problems, adapting a $2 \mathrm{D}$ mesh to reduce errors (adaptive analysis), and automatically remeshing large deformation analysis problems.

\section{Initial 2D Mesh Generation}

Automated meshing must not only significantly reduce time for the analyst, but it must also produce a high quality mesh. This implies that the analyst can control the element size, that the tements are well shaped, and that the process is robust.

Paving allows the user to maintain varying levels of control over the size of elements. This control ranges from the specification of a single desired element size for the entire mesh to the specification of a completely arbitrary sizing function over individual regions of the geometry. The transitioning capabilities are critical for this type of fine control.

In a qualitative sense, paving tends to produce a nice mesh with very square elements. Unfortunately, the quality of a mesh has historically been tied more to the analyst's opinion than to the actual goodness of the elements. As a more direct quantiative measure of the quality of the mesh, a distortion metric (Oddy et al., 1988) which calculates a distortion index for each element has been used. This metric is size and orientation indepeindent and combines effects of stretching and shearing of elements. With this measure, an element with a $2: 1$ aspect ratio and square corners (stretching) has an index of 1.0. Likewise a parallelogram shaped element with equal side lengths and a 65 
degree interior angle (shearing) has an index of 1.0. Typically, the average element distortion index in a paved mesh is around 0.1 with the maximum distortion index for any element being on the order of 1.0 .

Robustness, even more so then quality, is a difficult issue to address quantitatively. Response from the user community is our only measure. This technique has been in production use for over a year, bug reports are surprisingly scarce, and feedback from users has been very positive. There are some combinations of geometric and sizing constraints that cause problems for paving, sucl: as narrow regions where extreme transitions are required across the narrow portion of the region. These cases can usually be resolved with adjustments to the element size constraints.

\section{D Mesh Generation With Paving}

The generation of many 3D hexahedral meshes can be accomplished as combinations of various $2 \mathrm{D}$ surface meshes translal : $d$ or rotated through a volume. We have termed these $3 \mathrm{D}$ mesh portions which are generated from $2 \mathrm{D}$ meshes as being 2.5D. Although meshing $2.5 \mathrm{D}$ geometries involves a decomposition of the $3 \mathrm{D}$ part into these $2.5 \mathrm{D}$ pieces, this decomposition is significantly less work than the more standard practice of complete decomposition into 6 sided blocks for mapped mesh generation. Figure 6 shows two examples of $3 \mathrm{D}$ meshes generated using the paving algorithm and translations of the 2I) meshes.

We are currently developing a solid modeling based system to further simplify this decomposition and meshing of $3 \mathrm{D}$ geometries. The solid modeler will allow quick and unambiguous geometric definition by the user. We are implementing a number of operators that will ease the decomposition of this solid into appropriate pieces for meshing (by whatever meshing technique desired). We are also implementing a data modeling scheme that will allow adjacent pieces to share bounding faces (non-manifold cellular topology), thus eliminating the need for an equivalencing step combine seperately generated coincident nodes. This 

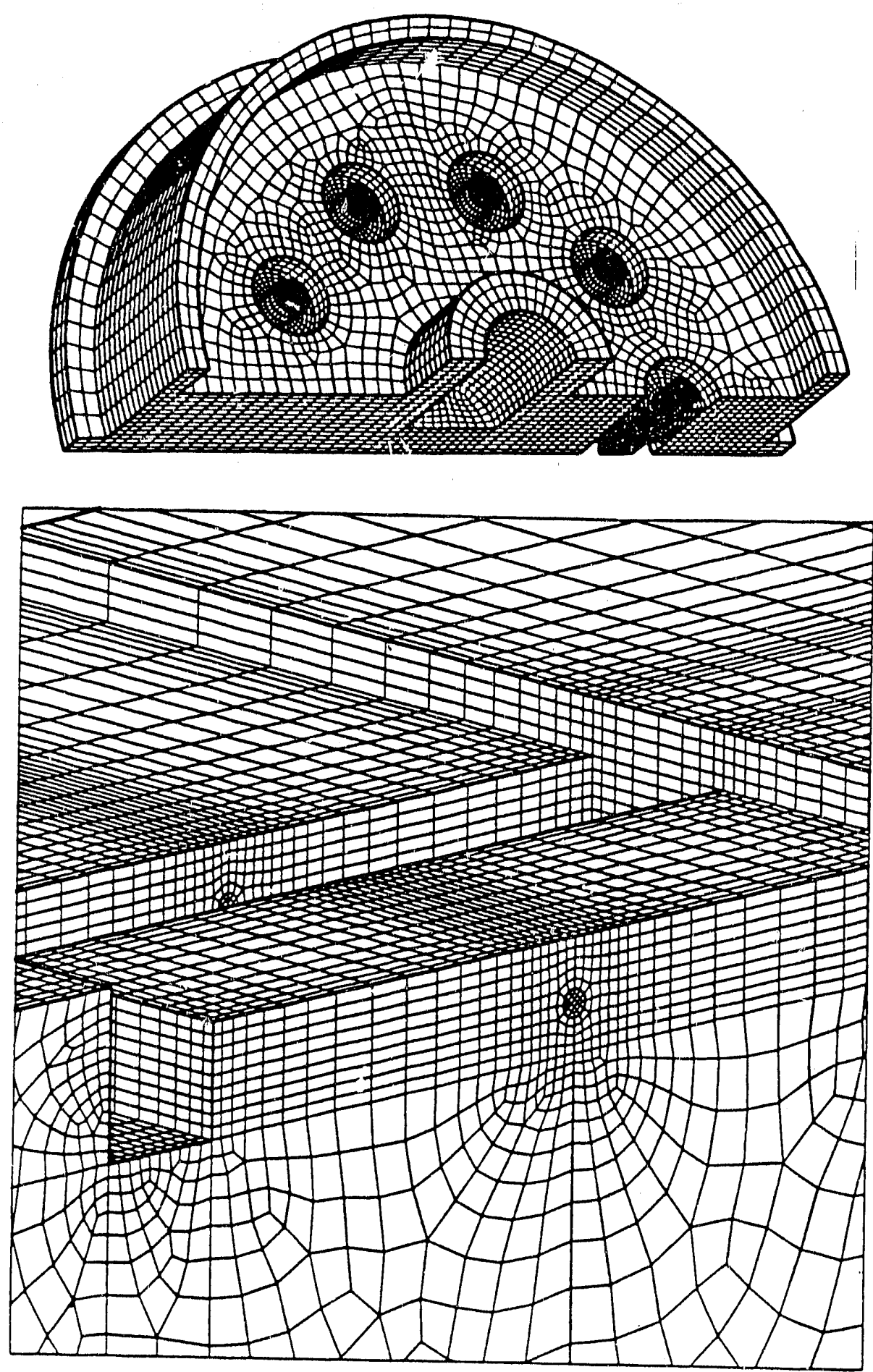

Figure 6: Example 3D Meshes Generated From 2D Paved Meshes 
system will lend itself well to the generation of $2.5 \mathrm{D}$ hexahedral meshes as well as the more general $3 D$ techniques described below.

\section{Adaptive Analysis Using Paving}

One of the most itcent applications of paving has been in adaptive analyses where equilibrium errors (Jung, 1990) in a finite element analysis are aulomatically reduced by iteratively meshing, solving, estimating errors, and then remeshing based on the error estimates (Blacker, Jung, and Witkowski, 1990). With this lechnique, a 2D finite element problem can be adaptively meshed to produce an answer within a desired error tolerance. Not only does this free the experienced analyst of tedious remeshing tasks, but it also safely opens some of the power of the finite element analysis technique to less-skilled users.

To use paving for adaptive analyses, the element size must be defined as a function of the error. We construct that function such that the desired mesh size is based on the error in, and size of elements in the existing mesh. Elenent sizes in regions with errors significantly higher than the target error are aggressively refined (reduction factors of 7.0 ) while element sizes in regions with low error relative to the target are conservatively expanded (expansion factors of 1.2).

As an example of the adaptive analysis capabilities, the symmetric plane strain structure shown in Figure 7 is analyzed. A pressure loading is applied to the interior cavity and a downward force applied to the lop arm. The deformed shape is shown in Figure 8. The initial and final meshes are shown in Figures 9 and 10 , respectively. The maximum discretization error for this problem was reduced by a factor of 20 as a result of this adaptive analysis process.

\section{Large Deformation Problem Remeshing}

We are currently developing a capability whereby large deformation analyses can be enhanced through the use of paving. This 


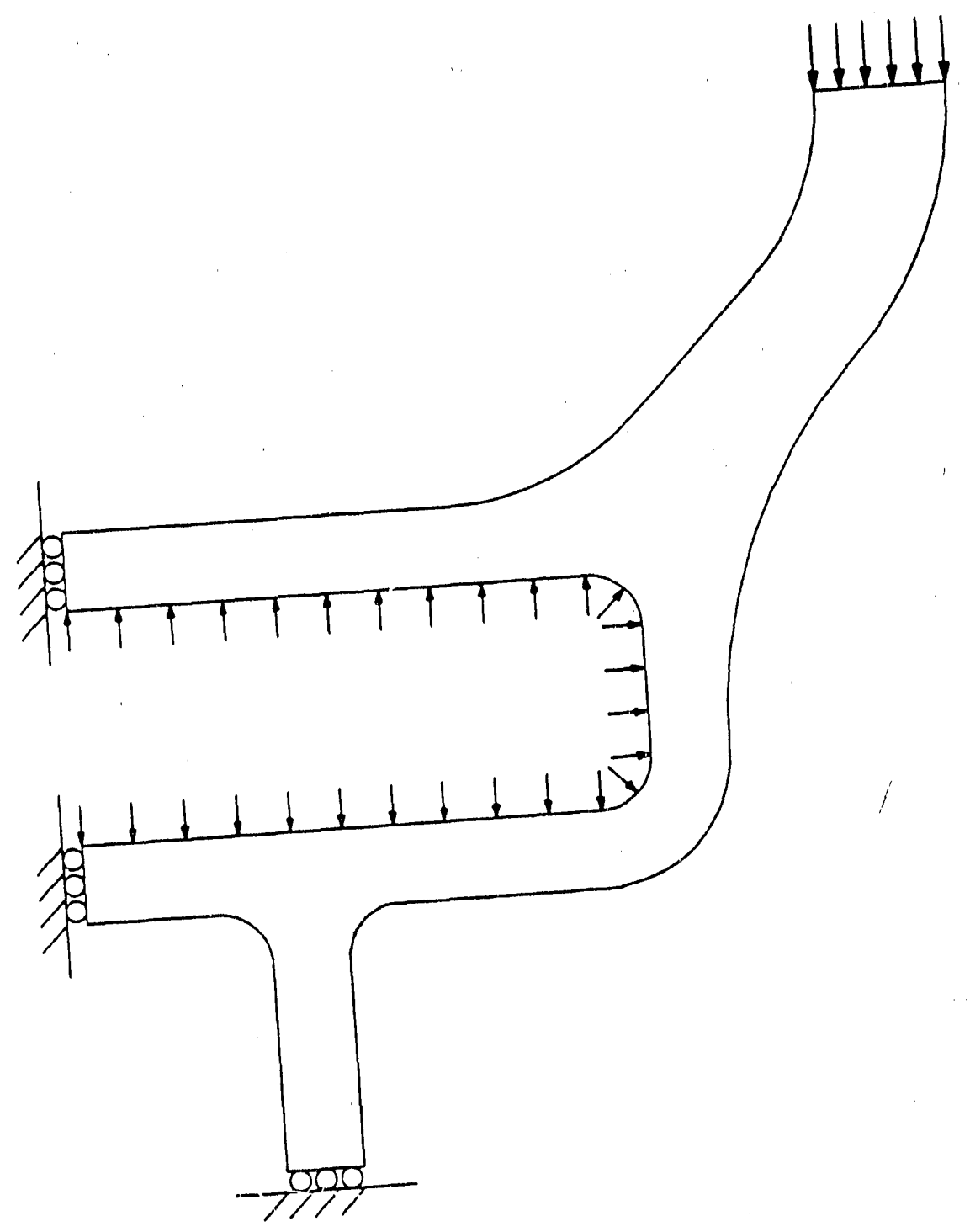

Figure 7: Adaptive Analysis Example Geometry 


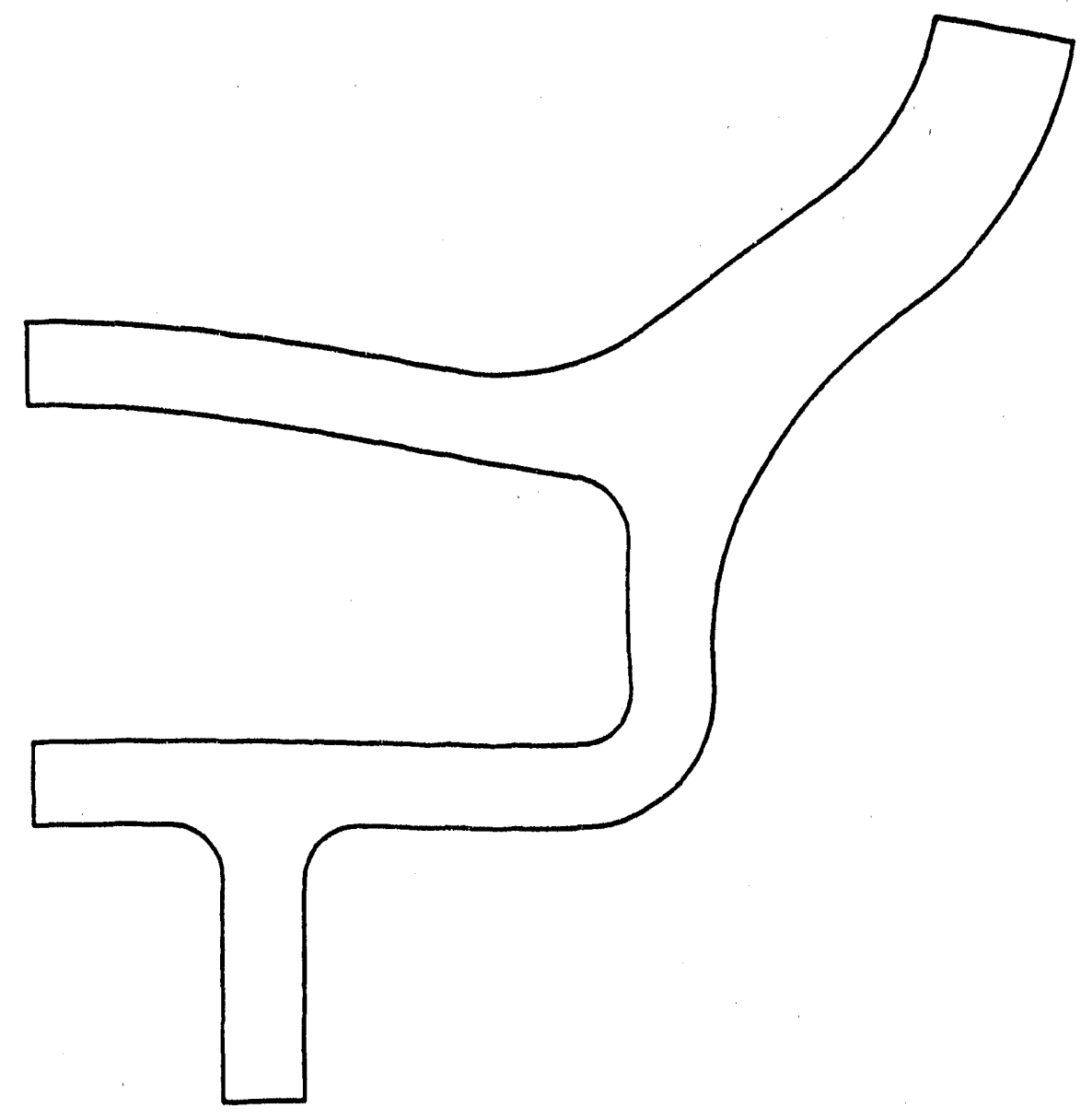

Figure 8: Adaptive Analysis Example Deforned Shape 


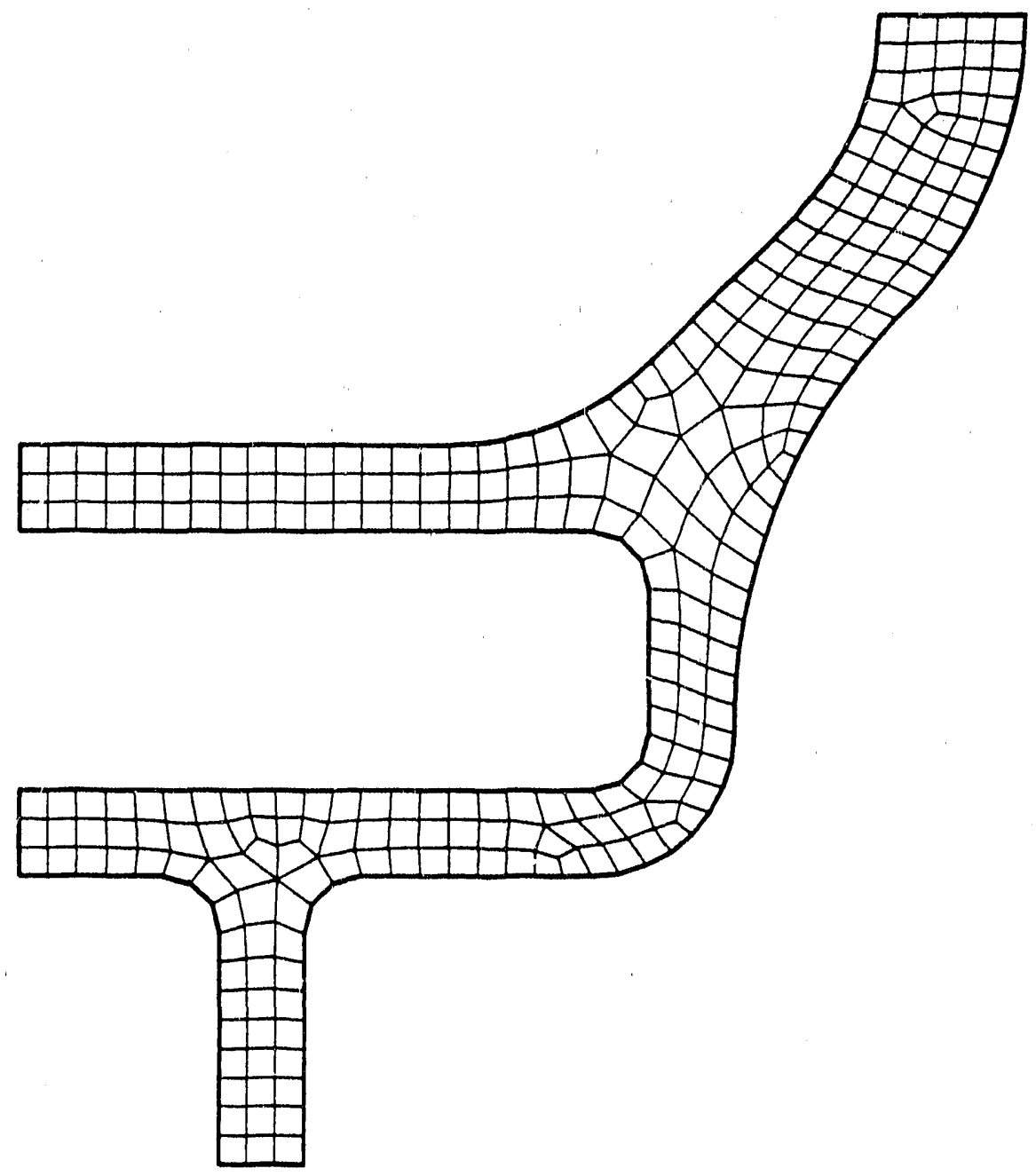

Figure 9: Initial Mesh for Adaptive Analysis Example 


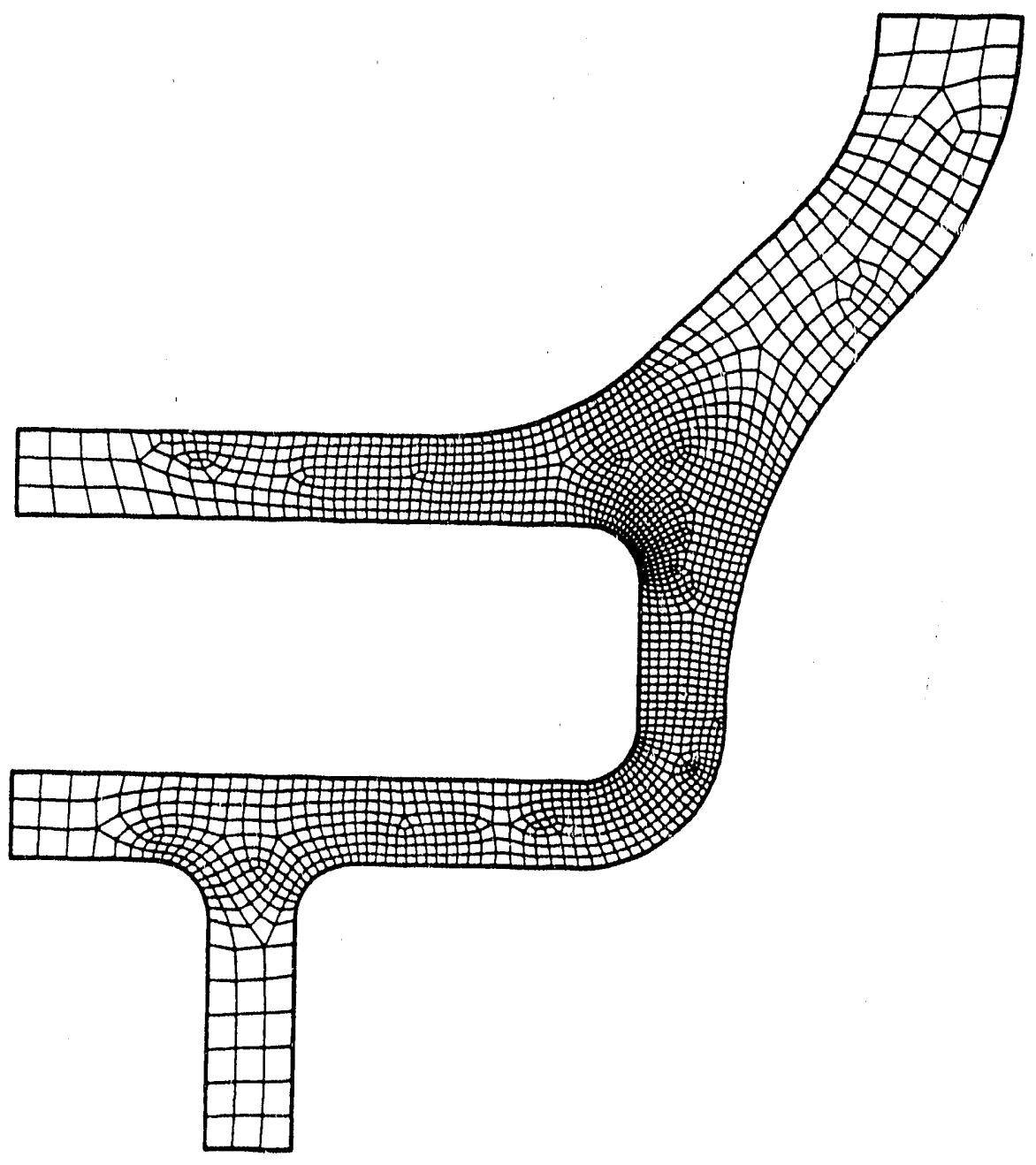

Figure 10: Final Mesh for Adaptive Analysis Example 
involves the generation of a new mesh based on the deformed geometry as defined by the exterior element edges, rather than the initial undeformed geometry. The analysis variables (stress, velocity, etc.) must then be mapped from the old mesh elements to the new elements. This approach of automatically generating a completely new mesh should prove a significant enhancement to existing techniques which simply smooth the existing mesh nodes in an attempt to improve mesh quality. 


\section{D Surface Meshing}

Current development is focusing on the extension of $2 \mathrm{D}$ paving to $3 \mathrm{D}$ all-quadrilateral surface meshing. To begin surface paving, a list of the boundary edges of the $3 \mathrm{D}$ trimmed surface is necessary. The edges of the face are discretized into nodes based on global (all edges) or specific (edge by edge) size requirements as specified by the user. These nodes form the boundary node list necessary for paving the geometris face. The paving lechnique is then used to form a valid quadrilateral mesh within this boundary. Care must be taken to insure that the nodes are placed on the surface as they are generated and that they remain on the surface during subsequent operations (e.g. smoothing). Surface paving will be useiul for general shell element generation in 3D as well as essential for the $3 \mathrm{D}$ solid meshing described below.

\section{D Solid Meshing}

A new research initiative is underway in which the paving technique is being extended to $3 \mathrm{D}$ all-hexahedral mesh generation. This $3 \mathrm{~V}$ extension, termed plastering, uses the same basic philosophy of discretizing the boundary of the geometry and then progressing inward from the boundary to form the entire mesh.

For this techuique, a boundary representation of the solid geometry is used. Each of the trimmed faces of the solid are first meshed with 3D paving as described above. This set of quadrilateral elements from paving now forms the initial plastering boundary in the same sense that the list of boundary nodes forms the initial paving boundary. These surface elements which form the plastering boundary are then used as elemental faces for the generation of a layer of hexahedral elements. Analogous to the addition of rows in paving, in plastering layers of elements are carefully constructed and added until the geometry has been filled with hexahedral elements.

'This research has progressed to the point that an all-hexahedral mesh can be generated for simple geometries using 3D plas- 
tering (Blacker, Stephenson, and Canann, 1990). More complex geometri.s are now being attempted. 


\section{Conclusions}

We have found the paving technique to significantly reduce costs associated with finite element analysis. The technique allows general 2D geometries to be meshed automatically, climinating much of the ledium of mesh generation and opening the technique for uoe by less skilled personnel. Its power has already been leveraged for a large class of $3 \mathrm{D}$ meshing problems (2.5D decomposable geometries). Improvements in the decomposition process will be realized as solid modeling engines are added to the meshing routines. Adaptive meshing using an error estima. tion tecinnique and paving can effectively reduce error and thus improve the reliability of the analyses being performed. The current 3D paving development will extend the paving algorithn to general 3D trimmed surface meshing and the $3 D$ plastering research also appears promising for generating a $3 \mathrm{D}$ all-hexahedral mesh within an arbitrary solid. 


\section{References}

Baehmanv, P. L., S. L. Witichen, M. S. Shepard, K. R. Grice, and M. A. Yerry, "Robust Geometricaly Based, Automalic Two-dimensional Mesh Generation", Int. J. for Num. Methods in Eng. 24, 1043-1078, 1987.

Blacker, T. D., J. Jung, and W. R. Witkowski, "An Adaptive Finite Element T'echnique Using Element Equilibrium and l'aving," ASME Winter Annual Mtg. Proc., 1990.

Blacker, T. D. and M. B. Stephenson, "Paving: A New Approach to Automated Quadrilateral Mesh Generation," SANDqu.0249, Sandia National Laboratories, 1990.

Blacker, T. D., M. B. Stephenson and S. Canann, "Plastering: A New Approach to Automated 3D Hexahedral Mesh Generation - Progress Report I," SAND89-2192, Sandia National Laboratories, 1990.

Joe, B. and R. B. Simpson, "Triangular Meshes for Regions of Complicated Shape", Int. J. for Num. Methods in Eng. 23, $751-7781986$.

Jung, J., "Finite Element Error Estimation and Adaptivity Based on Projected Stresses," SAND90-0416, Sandia National Laboratories, 1990.

Kela, A., R. Perruchio, and H. B. Voelker, "Toward Automatic Finite Element Analysis", Comp. Mech. in Eng. 5,11986 .

Oddy, A., J. Goldak, M. McDill, M. Bibby, "A Distortion Metric For lsoparametric Finite Elements", Transactions of the CSME, No. 38-CSME-32, Accession No. 2161, 1988.

Schoofs, A. J. G., L. H. T. M. Van Beukering and M. L. C. Sluiter, "A General Purpose 'Two-dimensional Mesh Generator", Advan. in Eng. Software 1-3, 131-136, 1979. 
Shepard, M. S. and M. A. Yerry, "Approaching the Automalic Generation of Finite Element Meshes", Comp. in Mech. Eng. 1, 49-56, 1983.

Wordenweber, B., "Finite-Element Analysis For The Naive User", Solid Modeling by Compulers, ed. M. S. Pickett and J. W. Boyse, Plenum Press, 81-102, 1984. 

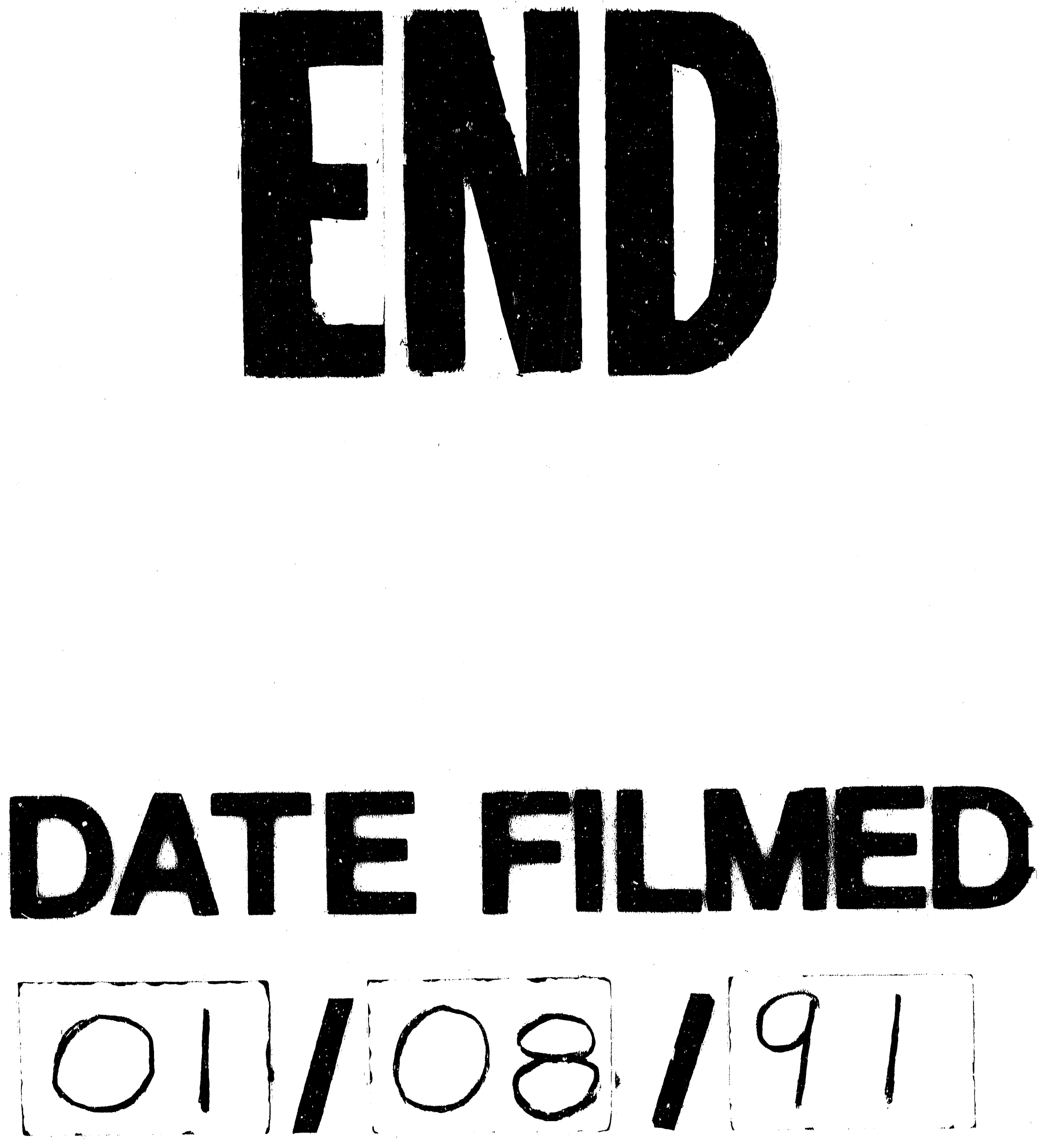